\title{
Taking the bull by the horns
}

Genetic analysis of domestic animals demonstrates the feasibility of marker-assisted selection of economically important traits and may foreshadow good things to come (other than better bacon) for human geneticists.

"MOLECULAR genetics has arrived on the farm", concluded James Womack in a Nature News and Views article ${ }^{1}$ late last year. So it has, there being several reasons behind the endeavour to understand the molecular genetics of domestic animals. Veterinarians wish to define the hereditary component of common diseases and thus eliminate them (negative selection); breeders aim to identify and exploit markers of economically important characteristics of their animals (positive selection); and geneticists can investigate genome organization and evolution by examining homologous sequences across species (comparative mapping).

Whereas comparative mapping requires extensive and precise genome maps from several species (see ref. 2 for review), isolating markers of diseases or desirable traits can, and is, progressing piecemeal. In the June issue of Nature Genetics, groups led by Michel Georges and Jay Hetzel ${ }^{3}$ describe a linkage study in which the autosomal polled locus (controlling horn development) is seen to segregate with two microsatellite markers on chromosome 1 in Bos taurus (a classification that includes most of the wellknown European cows). Previously it was only assumed that a polled locus was the main determinant of the horn phenotype ${ }^{4}$. Georges et al. confirm both the existence of the locus and its mode of inheritance, paving the way for generation of a physical map of the region and isolation of the gene. It can be assumed that, before the gene itself is isolated, closer markers (there is an estimated 13 per cent recombination rate between the two microsatellite markers and the polled locus) will be found allowing marker-based selection of the trait.

Why make a fuss about a rudimentary linkage study? Horns are generally considered undesirable (they present a risk of injury to both the animals and their handlers) and currently a surgical procedure is routinely used to de-horn cattle. But although the possibility of being able to

Also in this month's Nature Genetics: $\beta$-cGMP phosphodiesterase mutations in recessive retinitis pigmentosa; an artificial neo-organ for gene therapy to correct Sly syndrome; novel gene fusion event produces tumour-specific marker; and screening for unstable trinucleotide repeats. breed hornless animals is enticing, the real interest in this work is that it demonstrates the feasibility of marker-based selection in domestic animals.

Until now, breeders searching for an improved characteristic in their animals have had to embark on time-consuming breeding programmes. Despite the limitations of this approach, the economic potential of a slightly improved animal is such that extensive and elaborate schemes involving thousands of animals have been established. Molecular geneticists offer an alternative, and although breeders have shown some scepticism towards the new genetics impatience with traditional approaches (which can take 20-30 years to establish a new breed) has forced them to sit up and pay attention.

Four years ago the first bovine reference pedigrees (directly comparable to the indispensable human pedigrees produced by the Centre d'Etude du Polymorphisme Humain) were established by the Commonwealth Scientific and Industrial Research Organization in Queensland, Australia. This group and others (notably Genmark of Salt Lake City) started generating genetic markers. Their greatest challenge, the same as that of human geneticists, is to establish evenly spaced genome markers and use them to define singlegene traits and (ultimately) unravel the complicated genetics of multifactorial traits. Just as human geneticists have struggled with very sparse linkage maps, so too have animal geneticists, and with the publication of the first linkage studies that convincingly establish (distant) markers to single-gene traits ${ }^{3,5}$ they are reaping the rewards.

This work is now well advanced. The cytogeneticists who are able to identify the 30 , mostly acrocentric, chromosomes of the bovine genome are using in situ hybridization to map sequences. An international collaboration is working on a bovine genome map, and linkage maps for chromosomes 1 and 21, with markers spaced at 20 centimorgans, are now complete. This same collaboration has also established large full-sibling pedigrees to complement the existing reference pedigrees, and is pooling somatic cell hybrids. Animal breeders hope to be able to select characteristics such as improved milk production, better disease resistance, greater muscle mass and reduced fat content, and genetically to select for flavour and tenderness of meat. On the face of it these are daunting aims; nonetheless, certain breeds do produce more tender meat and some breeders would even claim to be able to differentiate between breeds on the basis of meat flavour.

The repercussions of this enterprise go further, f@r as domestic animal genes are identified and sequenced they will contribute to the data on comparative mapping. Indeed comparisons between sheep and cattle have shown that about half of the cattle markers may be useful in sheep studies (a not inconsiderable discovery, as very few markers are established in sheep linkage $\operatorname{maps}^{6}$ ). Furthermore, there is the prospect of producing a new generation of domestic animal models of human genetic diseases. Those massive breeding programmes, underway for many years, have thrown up a variety of unusual phenotypes. Although most such animals are slaughtered early in life, some are of great interest to the farming community (for instance, in contrast to the human condition, muscle hypertrophy in an animal reared for its meat is a very valuable characteristic). Occasionally, a particular phenotype will be recognized as bearing similarities to a human disease and the animal will be further investigated. Bovine models for Marfan's syndrome (for which no other animal model has been reported), Pompe's disease (glycogen storage disease II), osteogenesis imperfecta and Ehlers-Danlos syndrome have already been identified, and although there are drawbacks to having such a large animal as a model there are also some benefits - like humans, for instance, the cow has a nine-month gestation period, and the size of the animals means that there is no shortage of material for investigating fetal development.

It will be some time before complete comparative maps, well-characterized models or selection of mutifactorial traits become commonplace. But the paper by Georges et al. is one of those straws which show which way the wind is blowing and makes plain that these are worthwhile and realistic goals.

Adrian J. Ivinson

Adrian J. Ivinson is assistant editor of Nature Genetics.

\footnotetext{
1. Womack, J. E. Nature 360, 108-109 (1992)

2. O'Brien, S. etal. Nature Genet. 3, $103-112$ (1993).

3. Georges, M. etal. Nature Genet. 3, 206-210 (1993)

4. Long, C. R. \& Gregory, K. E. J. Hered. 69, 395 (1978)

5. Georges, M. et al. Proc. natn. Acad. Sci. U.S.A. 90 1058-1062 (1993).

6. Montgomery, G.W. et al. Nature Genet. (in the press).
} 